\title{
On the Transformation of Object-Oriented Conceptual Models to Logical Theories
}

\author{
Pieter Bekaert*, Bert Van Nuffelen, Maurice Bruynooghe, David Gilis, and \\ Marc Denecker \\ Katholieke Universiteit Leuven \\ Department of Computer Science \\ Celestijnenlaan 200A \\ 3001 Leuven, Belgium \\ \{pieter.bekaert, bert.vannuffelen, maurice.bruynooghe, david.gilis, \\ marc. denecker\}@cs.kuleuven . ac . be \\ http://www.cs.kuleuven.ac.be/ dtai/kt/btw/
}

\begin{abstract}
This paper describes a semi-automatic transformation from object-oriented conceptual models to logical theories. By associating a logical theory with a conceptual model, we are able to combine the best of both worlds. On one hand, the object-oriented software development paradigm is recognized to be well-suited to build maintainable and communicable conceptual models. On the other hand, the logical programming paradigm offers very powerful and semantically founded concepts to represent knowledge and the use of logical inference systems makes it possible to prototype solutions to computational tasks.

Since our method makes this mapping from conceptual models to logical theories traceable, dealing with the evolution of the problem domain and requirements becomes more manageable. Moreover a path is offered towards building prototypes for object-oriented conceptual models.
\end{abstract}

\section{Introduction}

Recently, there is a lot of research interest for providing formal semantics for Object-Oriented modeling languages, in particular for the Unified Modeling Language [16]. Most OO modeling languages lacked formal semantics, often introducing ambiguity and imprecision in the meaning of a model. Defining a transformation to another formalism is one approach to define semantics. For example, a translation from UML models to Z [6] has been investigated as part of the Precise UML project. Executable specifications, allowing validation and rapid prototyping, is another important issue of investigation [11]. It is however important to retain the qualities of good OO conceptual modeling, such as declarativity.

We believe that a mapping of conceptual models to logical theories, if done properly, gives both a firm semantics to the OO conceptual modeling language

\footnotetext{
* Research Assistant of the Fund for Scientific Research - Flanders (Belgium) (F.W.O.

- Vlaanderen)
} 
in question and creates an executable specification. The logic programming paradigm, in particular, offers very powerful and semantically founded concepts to represent knowledge about a problem domain. The construction and manipulation of the logical theories, however, can be a complex activity. OO analysis methods are better suited to build maintainable and communicable conceptual models of the problem domain.

A key quality of such a transformation is traceability. This offers an explicit connection between the conceptual model and the executable specification. The lack of traceability between different views of a system in development or maintenance creates a lot of problems and inconsistencies, especially when the requirements evolve. In a traceable process, changes to models and decisions are propagated in a manageable way.

Our approach starts with building a conceptual model with EROOS, an OO conceptual modeling method. This model then is transformed into an ID-Logic logical theory that forms the basis for prototyping the implementation of the software via the use of a logical inference system, the $\mathcal{A}$ system. In Sect. 2, we give an overview of the EROOS method for OO conceptual modeling and introduce an example model that will be used in the rest of the paper. In Sect. 3, ID-Logic is presented as a language for representing logical theories. Section 4 describes the transformation. Section 5 introduces some typical tasks and gives an outline of how they can be solved using the $\mathcal{A}$ system general solver. Finally, we conclude and set the directions for further research.

\section{Object-Oriented Conceptual Modeling in EROOS}

The ERCOS method (An Entity Relationship based Object-Oriented Specification method $[20,18,17])$ is an $\mathrm{OO}$ analysis method for building conceptual models (augmented with behavior). EROOS uses a notation with a formal and declarative nature. The modeling constructs introduced by the method offer strong guidance to the modeler, imposing a number of methodological rules. These restrict the expressivity of the modeling language, ruling out inferior ways to model reality. In this way, EROOS differs significantly from most other notations and methods such as UML [16] and RUP [12] or as in e.g. [14].

The example model used throughout the paper represents a simplified part of an educational resource management system [4]. The purpose of the system is amongst others to administer which teaching assistant has to teach which exercise sessions. The graphical representation of the model is given in Fig. 1 .

An EROOS conceptual model introduces a number of classes corresponding with the concepts that are identified in the problem domain, e.g. COURSE, SESSION, EXERCISE TEACHER. A class can be refined at most once with a (unary or binary) relation involving (one or two) participating classes. ${ }^{1}$ This introduces an existential dependence for each object of the refined class upon a single object

\footnotetext{
${ }^{1}$ This methodological rule is a driving force in finding and organizing classes, leading to better models.
} 


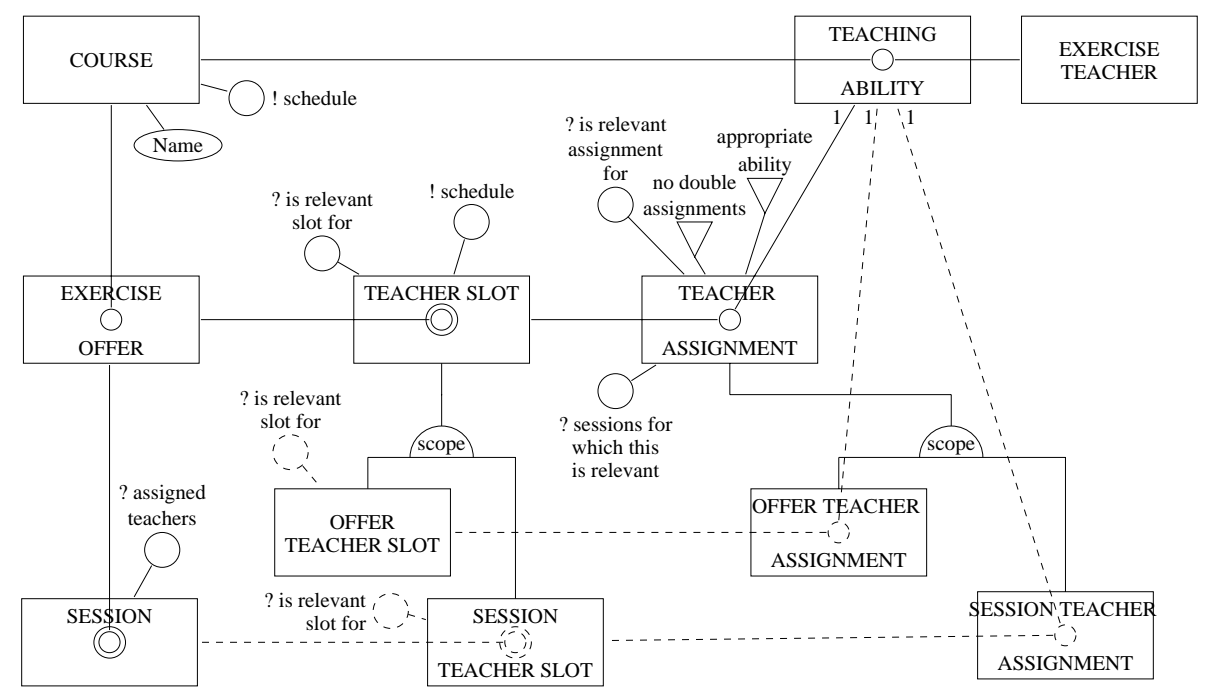

Fig. 1. An ERCOS conceptual model for an exercise teacher assignment system

of each participating class. A relationship is thus reified, unlike in most other methods, where relations or associations are modeled between classes as such.

Relations are represented by means of a circle in the refined class, with lines towards the participating classes. A single circle indicates a multiplicity restricted to one duplicate: e.g. at most one teaching ability can exist having the same participant tuple, i.e. the same exercise teacher and the same course. Unrestricted multiplicity is indicated with two circles (e.g. TEACHER SLOT). A "1" at the end of a participant line of a binary relation denotes a restricted connectivity: e.g. given a teacher slot, this object can be related to at most one teaching ability through teacher assignments.

Attributes can be defined for classes, introducing an existential dependence for their objects upon a value of a given domain. In the example, each course must have a name with the domain COURSE NAME as type.

EROOS emphasizes the formal specification of all constraints observable in the problem domain [19]. This can be done implicitly through the semantics of the constructs (e.g. existential dependence) or integrated in the definition of model elements (e.g. multiplicity and connectivity of relations). If not possible otherwise, explicit constraints can be specified on top of one or more classes, introducing a universally quantified logical assertion. The population of a model must at any instance of time obey all constraints.

Explicit constraints are represented by means of triangles connected to the classes to which they apply. The constraint appropriate ability states that for each teacher assignment, the course upon which the participating teaching ability depends must be the same as the course that is reached via the teacher slot and exercise offer. The constraint no double assignments states that the same 
exercise teacher cannot be assigned more than once to teach a certain session (via different teacher slots).

Building class hierarchies using generalization/specialization is an important aspect in OO conceptual modeling. In EROOS every characteristic of a generalization class is inherited in a specialization class, introducing an is a relationship and thus supporting polymorphic reasoning. Characteristics can be strengthened in specialization classes. Specialization classes are always grouped in a (mathematical) partition of the generalization class, turning the latter into an abstract class. EROOS supports multiple specialization through the definition of multiple orthogonal partitions [2].

A partition is indicated using a half circle. Dashed lines are used to represent that inherited characteristics are strengthened. In the model, two partitions are introduced. They model that a teacher slot, and consequently a teacher assign$m e n t^{2}$ can exist for the entire exercise offer (and thus for all it's sessions) or for a particular session. Note that these slots and assignments are additive.

Queries are defined for modeling derived information. They are denoted using circles with question marks, connected to the class on which they are defined. Queries can be applied to individual objects of this class. The query assigned teachers specifies which teachers teach a given session in terms of the other queries. (The queries with a name ending with "for" take a session as an argument.)

The evolution of the state in the problem domain is modeled by the occurrence of events upon objects. The specification of these events is part of an EROOS model. Events have a transactional, declarative nature. The schedule events in the figure, create assignments for all resp. a given vacant slot for a course. This can be specified elegantly using non-determinism [3]. In this paper, we will not describe the formalism for specifying queries, events and constraints.

Semantics for EROOS conceptual models, including non-deterministic behavior, have been defined in terms of populations and population evolutions, using set-theory and logic [3].

\section{Logical Theories}

\subsection{The Language and its Semantics}

Inductive Definition Logic (ID-Logic [7, 8]) is an integration of first order logic and Logic Programming ${ }^{3}$, interpreting the logic program as a non-monotone inductive definition. An ID-Logic theory is defined as a pair $(\mathcal{D}, \mathcal{F})$ where $\mathcal{F}$ is a set of first order statements, expressing true assertions of the domain of discourse. $\mathcal{D}$ is a logic program and represents a (non-monotone) inductive definition of a subset of the predicates. ${ }^{4}$ These are the defined predicates. Only defined predicates

\footnotetext{
${ }^{2}$ This second partition is not strictly needed in the simplified model, but is kept for illustrative purposes.

${ }^{3}$ Consequently ID-Logic generalizes all subclasses of Logic Programming, e.g. datalog.

${ }^{4}$ In the context of this paper, an ID-Logic theory contains only one definition (the logic program). In general [7], it can contain an arbitrary number of independent definitions.
} 
appear in rule heads. The other predicates are called open. Their interpretation is free.

The semantics of an ID-Logic theory are given by an interpretation $\mathrm{M}$ of $\mathcal{D}$ satisfying the following conditions. Let $M_{O}$ be an arbitrary two-valued interpretation for the open predicates in $\mathcal{D}$, fixing their meaning. This interpretation $M_{O}$ turns $\mathcal{D}$ in a standard logic program. The unique two-valued well-founded model $^{5}[21]$ of this resulting logic program is $\mathrm{M}$. M is only a model of the whole ID-Logic theory if it also is a model for $\mathcal{F}$.

As a consequence, the formulas in $\mathcal{F}$ constrain the possible interpretations of the open predicates.

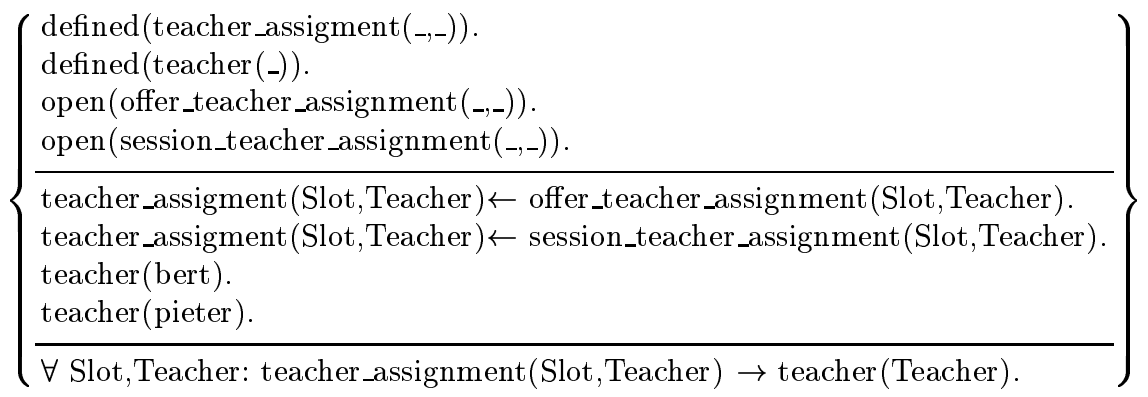

Fig. 2. An ID-Logic theory

Figure 2 shows an example of an ID-Logic theory. For readibility, the different parts are separated. The distinction between defined and open predicates is made explicit by the declarations in the upper part. In the middle, rules are given for the defined predicates. One is given in terms of other predicates and the other as a full enumeration. The bottom part contains the FOL formula that the second argument of teacher_assigment/2 must be a teacher. Remark that this theory is not the result of the transformation that will be defined later on.

\subsection{Computing Models using the Asystem}

Given an ID-Logic theory, a basic computational task is to find an interpretation $\Delta$ for the open predicates that is a solution. (This $\Delta$ can be specified by the set of atoms that are true in the interpretation, the atoms outside the set $\Delta$ are false.) This task is known as abduction and is studied in abductive logic programming [10]. However, an ID-Logic theory is also a generalization of standard logic programming. There, the basic computational task is deduction: Given a query $\leftarrow Q$ (with $Q$ a conjunction of literals), the task is to find a substitution $\sigma$ such that $Q \sigma$ is true in the unique model of the program.

The Asystem $[13,1]$ is able to combine both computational tasks, because it is an implementation of the SLDNFA procedure [9]. That SLDNFA procedure

\footnotetext{
${ }^{5}$ This is exactly the least Herbrand model in the case of a definite program.
} 
is a sound and complete abductive extension of the SLDNF procedure that is at the basis of any implementation of logic programming. Given an ID-Logic theory $(\mathcal{D}, \mathcal{F})$ and a query $\leftarrow Q$, SLDNFA computes a $\Delta$ and a substitution $\sigma$ (if they exist, otherwise it fails) such that (1) the well-founded model of $(\mathcal{D} \cup \Delta)$ is two-valued and (2) $Q \sigma$ and $\mathcal{F}$ are true in the well-founded model of $(\mathcal{D} \cup \Delta)$.

The $\mathcal{A}$ system has been used in a number of experiments [13], including computationally hard problems like AI-planning.

\subsection{Extensions of ID-Logic}

In the above discussion, we have formulated a basic version of ID-Logic and omitted a number of aspects which are of minor importance for the understanding of this paper. Besides the formulated core, ID-Logic is extended with a number of features that allow its users to express certain knowledge in a more concise and more readable way. Two features we make use of are type declarations and function declarations. These declarations enhance the readability of the language and they are exploited by the solvers to optimize the reasoning process.

In ID-Logic, a type declaration introduces a unary predicate. Figure 3 shows the unary predicate course/1. The actual definition of the predicate is as any defined predicate and belongs to the $\mathcal{D}$ part of the theory. It can be defined by an enumeration of facts, in terms of other predicates or declared open.

$$
\left\{\begin{array}{l}
\text { type course. } \\
\text { function number_of_attendees(course):int. } \\
\text { predicate follows_course(student,course). }
\end{array}\right\}
$$

Fig. 3. Type and function declarations in ID-Logic

Figure 3 also declares a function. On one hand, a function introduces an alternative notation for a predicate, here a predicate number_of_attendees $/ 2$, on the other hand it also introduces a formula in $\mathcal{F}$, namely the functional dependence between the arguments of the function and its result. It also introduces a formula restricting the domain and range of the function to the declared types. Also predicate arguments can be restricted to a declared type as the example shows.

Remark that both extensions can be compiled away to an equivalent type and function free theory. It is this theory that the Asystem will take as input.

\section{Transformation of EROOS Conceptual Models to ID-Logic Theories}

In this section we describe the transformation of EROSS conceptual models to ID-Logic theories. A tool has been developed that performs a large part of this transformation automatically. 
EROOS conceptual models are built using a conceptually rich set of constructs. In the process of the transformation to ID-Logic, we must define how to represent them in terms of definitions and FOL formulae. The theory that results is restricted to the structure of the problem domain, and does not contain information about the actual objects that are present. Neither does it declare predicates as defined or open. These aspects are only introduced in the context of tasks (see Sect. 5).

The EROOS events are currently not taken into account in the transformation, since the approach in logical programming for behavior is significantly different. The relation between both approaches is under investigation.

The transformation is presented in an informal way and illustrated in the context of the example introduced in Sect. 2. The transformation of all concepts is however clearly described and covered by the example.

\subsection{Transformation of Classes and Domains}

For each class in the conceptual model, a type is defined in the theory. This introduces a unary predicate that in a certain state of the system evaluates to true for those atoms that actually represent an object of that class. In the same way, types are defined for the domains of the attributes introduced in the conceptual model. Figure 4 shows the types generated for the conceptual model in Fig. $1{ }^{6}$ To reduce the length of the lines, we abbreviated the symbol names.

$$
\left\{\begin{array}{l}
\text { type courseName. } \\
\text { type course. } \\
\text { type session. } \\
\text { type teachAbil. } \\
\text { type teachSlot. } \\
\text { type offTeachSlot. } \\
\ldots
\end{array}\right\}
$$

Fig. 4. The ID-Logic theory: types

The type system introduced in Sect. 3, is very simple, allowing us to explicitly state the properties of the type system of EROOS (see Sect. 4.3).

\subsection{Transformation of Relations and Attributes}

Each refinement of a class with a relation gives rise to one or two functions (for unary resp. binary relations) expressing the existential dependences introduced by the refinement. A function is also introduced for each attribute. The domain of these functions is the type for the class that is refined with the relation or decorated with the attribute. The range is the type for the participating class resp. the domain of the attribute. The functions are shown in Fig. 5.

\footnotetext{
${ }^{6}$ We will only present some partial fragments. The full theory can be found in [5].
} 


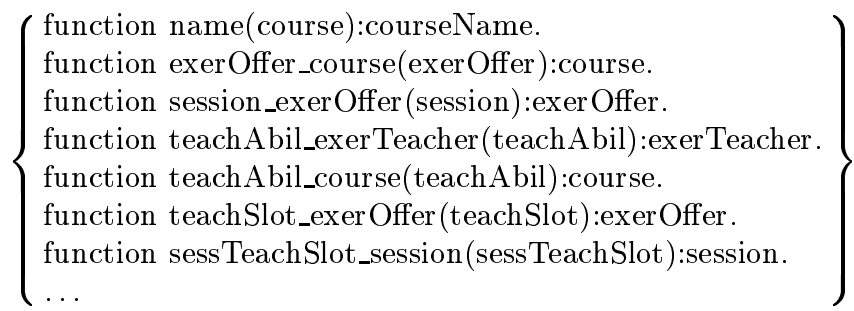

Fig. 5. The ID-Logic theory: functions for existential dependences

For binary relations, an alternative would be to introduce a ternary predicate instead of two functions. We would then need to impose functional dependences explicitly. The current representation is closer to EROOS.

The multiplicity and connectivity constraints integrated in the definition of the relation, are also added to the theory. This is shown in Fig. 6.

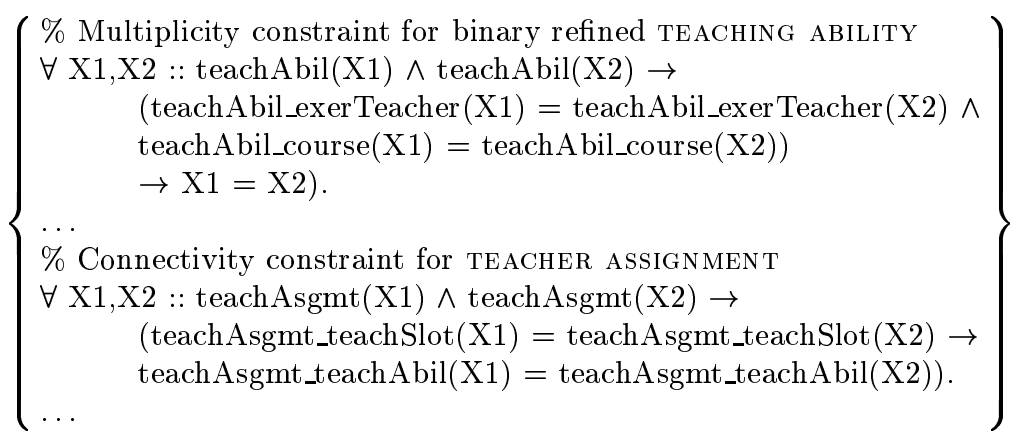

Fig. 6. The ID-Logic theory: integrated constraints for relations

\subsection{Transformation of Generalization/Specialization}

The primary characteristic of Generalization/Specialization, is that it introduces an is a relationship between the objects of a specialization class and a generalization class. This is also indicated in the ID-Logic theory. In EROOS however, generalization/specialization is a rich concept, that introduces a lot more. In our approach, we fully transform each class in isolation, and then relate them in the theory. An alternative is to define all generalization classes in terms of their specializations. The transformation presented below equally applies to multiple generalization/specialization hierarchies, as elaborated in [4]. 
Partitioning Constraints. As explained in Sect. 2, specialization classes are grouped in mathematical partitions of generalization classes. The implicit disjointness and completeness constraints are made explicit in the theory. This is shown, together with the is a relationship, in Fig. 7.

$$
\left\{\begin{array}{l}
\forall \mathrm{X}:: \operatorname{offTeachSlot}(\mathrm{X}) \rightarrow \text { teachSlot }(\mathrm{X}) . \\
\forall \mathrm{X}:: \operatorname{sessTeachSlot}(\mathrm{X}) \rightarrow \text { teachSlot }(\mathrm{X}) . \\
\forall \mathrm{X}:: \operatorname{teachSlot}(\mathrm{X}) \rightarrow(\operatorname{offTeachSlot}(\mathrm{X}) \vee \operatorname{sessTeachSlot}(\mathrm{X})) . \\
\forall \mathrm{X}:: \neg(\operatorname{offTeachSlot}(\mathrm{X}) \wedge \operatorname{sessTeachSlot}(\mathrm{X})) .
\end{array}\right\}
$$

Fig. 7. The ID-Logic theory: the "is a" relationship, completeness and disjointness for the partition scope of TEACHER SLOT

Finally, an object in EROOS can only belong directly to a single mostspecialized class. In other words, the populations of each couple of concrete classes is disjoint. Due to the limitations on the form of the class hierarchy, this is equivalent to stating that each couple of most general classes is disjoint, as done in Fig. 8.

$$
\left\{\begin{array}{l}
\forall X:: \neg(\operatorname{course}(\mathrm{X}) \wedge \text { exerOffer }(\mathrm{X})) . \\
\forall \mathrm{X}:: \neg(\operatorname{course}(\mathrm{X}) \wedge \operatorname{session}(\mathrm{X})) . \\
\ldots
\end{array}\right\}
$$

Fig. 8. The ID-Logic theory: each couple of most general classes is disjoint

Strengthening. The notion of specialization incorporates the very powerful possibility of strengthening characteristics of a generalization class at the level of the specialization class. This is done in the example for the refinement of classes with a relation. In the previous paragraph, functions and formulae were generated for all refined classes, independent of generalization/specialization and strengthening. The functions generated for the strengthened definition of the relation in the specialization class, must be related with those for the relation in the generalization class. Since objects of the specialization class must be interpretable as objects of the generalization class(es). This relation is defined in Fig. 9. The first formula, states that the participating teaching ability for a session teacher slot through the function at the specialized level must be the same as through the function at the generalized level. This is an unstrengthened participation, but the formula is needed. The second formula essentially does the same for the other participant. However, the range of the function introduced at the specialized level is restricted to a specialization of TEACHER SLOT (cfr. 


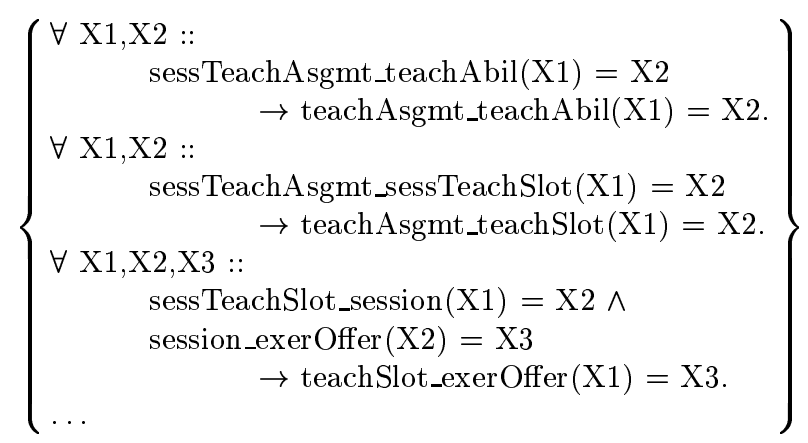

Fig. 9. The ID-Logic theory: strengthening of relations

Fig. 5). The third formula is more complicated. Here an additional link is inserted in the chain of existential dependence. The formula states that the function composition at the specialized level is equal to the function at the generalized level. Notice that in these formulae, no explicit domain is set for the variables of the universal quantification. The equations will be false for those X's out of range, thus not intervening with the truth of the formula.

\subsection{Transforming Explicit Constraints}

Since the formalism of EROOS is based on set theory and first order logic, we expect this transformation to pose no conceptual problems. It is however not yet worked out and implemented.

A possible formulation of a formula for the constraint appropriate ability on TEACHER ASSIGNMENT is straight-forward from its specification in EROOS and is given in Fig. 10 .

$$
\left\{\begin{array}{l}
\forall \mathrm{X}:: \text { teachAsgmt }(\mathrm{X}) \rightarrow \\
\text { exerOffer_course }(\text { techSlot_exerOffer(teachAsgmt_teachSlot }(\mathrm{X})))= \\
\text { teachAbil_course }(\text { teachAsgmt_teachAbil }(\mathrm{X})) .
\end{array}\right\}
$$

Fig. 10. The ID-Logic theory: The constraint appropriate ability on TEACHER ASSIGNMENT

\subsection{Transforming EROOS Queries}

The definition of a deterministic query in an EROOS model introduces information that can be derived from the current population by applying the query (with arguments) to an object. EROOS queries do not have preconditions and are thus 
always defined. In the ID-Logic theory, a deterministic, single-valued EROOS query can be represented by means of a function, with as domain the Cartesian product of the type introduced for the class of objects the query can be applied to and the types for the arguments, and with as range the type of the result. A query returning a set gives rise to a predicate. Figure 11 outlines the predicate generated for the query assigned teachers on SESSION. As for constraints, queries

$$
\left\{\frac{\frac{\text { predicate assigned_teachers(session,exerTeacher). }}{\text { defined assigned_teachers(_,_-). }}}{\text { assigned_teachers(Session,ExerTeacher }) \leftarrow \ldots}\right\}
$$

Fig. 11. The ID-Logic theory: The query assigned teachers on SESSION

are not yet dealt with automatically.

A conceptual model with recursive queries can introduce problems, since the semantic equivalence between the EROOS query and a definition in the theory is still to be investigated in that context. Non-deterministic queries also need to be further investigated.

For the full specification of the queries in the example, we refer to a forthcoming technical report [5].

\section{Solving Tasks}

The above transformation is the first step towards a prototyping system. This section shows the use of the $\mathcal{A}$ system to compute solutions for a given task. We present it by means of two important classes of tasks: namely database querying and scheduling. Traditionally, solutions for these applications use very different techniques.

\subsection{General Notes on Tasks}

Before presenting our task representations, we have to refine the view depicted above on the input of the Asystem. In OO modeling the distinction between an actual instance of the conceptual model (the population) and the conceptual model itself is commonly made. In a logical context this distinction is not always made. However in the context of deductive databases, it corresponds to the distinction between the extentional database (EDB) and the intentional database (IDB). In ID-Logic this EDB has to be provided for each task. In what follows, we represent it by $\mathcal{P}$ as it corresponds to the population of the conceptual model.

The description of a task consists on one hand of the theory $(\mathcal{D}, \mathcal{F})$, the population $\mathcal{P}$ and the set $\mathcal{O}$ containing the open predicates. On the other hand it consists of the query $\mathrm{Q}$ that triggers the computation. We define the triple 
$(\mathcal{D} \cup \mathcal{P}, \mathcal{F}, \mathcal{O})$ as the representation for the theory which is given to the $\mathcal{A}$ system to resolve the task.

To illustrate the reasoning process, we use the following population $\mathcal{P}$. The unmentioned classes are assumed to be empty. Also we have ignored the partitioning in the conceptual model to simplify the example population.

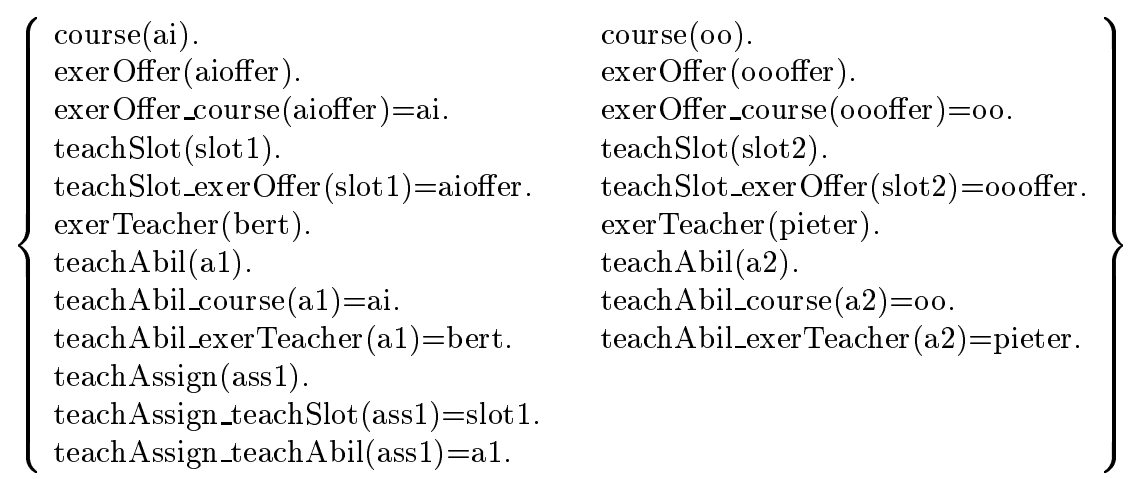

Furthermore when referring to $\mathcal{D}$ and $\mathcal{F}$ from now on, the result from the above transformation applied on the example conceptual model is meant.

\subsection{Database Querying}

A good characterization of this class of tasks is that of posing queries over a fully known database. In our formulation the population $\mathcal{P}$ is the database.

E.g. for the query: who teaches the exercises of course ai in our example, the answer is given by resolving the query assigned_teachers (ai,Teacher) in the context of the theory $(\mathcal{D} \cup \mathcal{P}, \mathcal{F}, \emptyset)$. Here, the $\mathcal{A}$ system will return the answer substitution $\sigma=\{$ Teacher/bert $\}$ and an empty $\Delta$ as answer.

Note that no open predicates exists (the set $\mathcal{O}$ is empty) because the database is complete and no hypotheses should be made about any of the predicates. This reduces the inference process of the $\mathcal{A}$ system to deductive reasoning.

\subsection{Scheduling}

This class differs from database querying in different points. Mainly because the expected answer is not a simple substitution for the query, but a set of objects and their dependences.

Let us explain this by example: suppose that we are looking for an assignment of teachers to exercises in the context of the population $\mathcal{P} . \mathcal{P}$ contains already a partial assigment: namely bert for slot 1 , however slot 2 is still vacant. Then an answer to the scheduling task is an extension of the set of teacher Assignments (and its dependences) that will fill up this gap.

In order to allow the system to generate this set, the expected answer predicates are declared open. For our example, the set $\mathcal{O}$ contains \{teachAsgmt(_), teachAsgmt_teachAbil(_,_), teachAsgmt_teachSlot(_,_-) $\}^{7}$.

\footnotetext{
${ }^{7}$ The partial information about these predicates in the population is now part of $\mathcal{F}$.
} 
Secondly for this task, we have to add the requirement that each slot must have a teacher assigned. This constraint is part of the scheduling event and does not appear in the conceptual model (and consequently in the derived theory), since it is not to be fulfilled during the entire system life-cycle. But we have to take it into account, because it enforces the search for a schedule. Formally the requirement is $\forall X::$ teachSlot $(X) \rightarrow \exists Y$ :teachAsgmt_teachSlot $(X)=Y$.

Bringing everything together we obtain the input theory $(\mathcal{D} \cup \mathcal{P}, \mathcal{F} \cup\{\forall X:$ : teachSlot $(X) \rightarrow \exists Y$ : teachAsgmt_teachSlot $(X)=Y\}, \mathcal{O})$. The query for a scheduling task is typically true because the interest is in the set teacher Assignments.

Note that solving this problem cannot be done by deductive reasoning, but needs abductive reasoning. The computed answer is here an empty answer substitution and the set $\Delta=\{$ teachAsgmt(ID1), teachAsgmt_teachSlot(ID1)=slot2, teachAsgmt_teachAbil(ID1)=a2 $\}$ in which ID1 is a new object identifier belonging to the set teacher Assignments.

\subsection{Some Remarks}

The presented approach contains all the ingredients needed to build a prototype system out of it. It is a very flexible approach to describe a task and is capable to deal in a simple, uniform way with different kinds of problems (namely by declaring open and defined predicates). Hence making it well suited to be the kernel of a prototype system.

As far as efficiency is concerned, the current prototype executes the whole transformed program, a rather time consuming task. Obviously, when knowing which predicates are defined and open and the population, many constraints could be simplified. We expect this to be possible with off the shelve logic program specializers such as ECCE [15] but that has not yet been explored.

\section{Conclusion and Future Work}

In this paper we have presented a transformation from object-oriented conceptual models in EROOS to logical theories in ID-Logic. This is a first step towards a method that provides a modeling tool for logical theories. At the same time the transformation is a first step towards a complete formal semantics for EROOS conceptual models in a well established formalism. By following a transformative approach, we try to build a method that supports the evolutive aspects of the software development process. Moreover this approach makes it possible to build a prototype of the system, and even to perform computational tasks in the context of the generated theory. This has been worked out for two important classes of tasks: database querying and scheduling.

The work presented here is only a beginning. First of all, the transformation is only partial. Certain concepts of the EROOS method, e.g. the archive and object life-cycles, are left out. Explicit constraints and queries are not yet 
translated automatically and events are not yet taken into account at all. Nondeterministic constructs in EROOS allow complex planning functionalities to be specified in an elegant way [3]. Important work is to be done to integrate the EROOS approach to modeling behavior using queries and events with the (Abductive) Logic Programming approach using tasks. We expect that this will result in a deeper understanding of both paradigms.

Using theorem provers, we can verify if the given conceptual model entails a certain property, starting from the generated logical theory. An interesting application of this approach consists in proving for each class, that a non-empty population can exist. If not possible, this may for instance indicate malformed (multiple) generalization/specialization hierarchies.

The transformation generates a theory that is entirely tailored to the conceptual model at hand. An alternative approach would be to see a conceptual model as an instance of a meta-model. If for this meta-model a corresponding meta-theory is made in logic, it is possible to transform a conceptual model to an instantiation of this meta-theory. All semantics of the EROOS constructs are then expressed once at the level of this meta-theory. Moreover in logical knowledge representation, meta-theories are built for specific types of knowledge and reasoning patterns. Bringing this back to OO conceptual modeling can introduce interesting modeling constructs and patterns.

\section{References}

[1] The Asystem. Obtainable via www.cs.kuleuven.ac.be/ dtai/kt/systems-E.shtml.

[2] Pieter Bekaert, Geert Delanote, Frank Devos, and Eric Steegmans. Specialization/Generalization in Object-Oriented Analysis: Strengthening and Multiple Partitioning. In Workshop MASPEGHI: MAnaging SPEcialization/Generalization HIerarchies, OOIS 2002, September 2002.

[3] Pieter Bekaert and Eric Steegmans. Non-Determinism in Conceptual Models. In Proceedings of the Tenth OOPSLA Workshop on Behavioral Semantics, OOPSLA'01, October 2001.

[4] Pieter Bekaert, Bert Van Nuffelen, Maurice Bruynooghe, David Gilis, and Marc Denecker. Specifying An Educational Resource Management System: A Transformative Approach. Report, Department of Computer Science, K.U.Leuven, Leuven, Belgium, in preparation, draft at http://www.cs.kuleuven.ac.be/ dtai/kt/btw/.

[5] Pieter Bekaert, Bert Van Nuffelen, Maurice Bruynooghe, David Gilis, and Marc Denecker. Transforming EROOS Conceptual Models to ID-Logic Theories: A Case Study. Report, Department of Computer Science, K.U.Leuven, Leuven, Belgium, in preparation, draft at http://www.cs.kuleuven.ac.be/ dtai/kt/btw/.

[6] Jean-Michel Bruel and Robert B. France. Transforming UML models to formal specifications. In Pierre-Alain Muller and Jean Bézivin, editors, Proc. International Conference on the Unified Modeling Language (UML): Beyond the Notation. Springer-Verlag, 1998.

[7] Marc Denecker. Extending classical logic with inductive definitions. In John Lloyd, Veronica Dahl, Ulrich Furbach, Manfred Kerber, Kung-Kiu Lau, Catuscia Palamidessi, Luis Moniz Pereira, Yehoshua Sagiv, and Peter J. Stuckey, editors, 
Computational Logic - CL 2000, First International Conference, London, UK, July 2000, Proceedings, volume 1861 of Lecture Notes in Artificial Intelligence, pages 703-717. Springer, 2000.

[8] Marc Denecker, Maurice Bruynooghe, and Victor Marek. Logic programming revisited: logic programs as inductive definitions. ACM Transactions on Computational Logic, 2(4):623-654, October 2001.

[9] Marc Denecker and Danny De Schreye. SLDNFA: an abductive procedure for normal abductive programs. Journal of Logic Programming, 34(2):111-167, February 1998.

[10] Marc Denecker and A.C. Kakas, editors. Special Issue : Abductive Logic Programming. Elsevier, North-Holland, 2000. Special issue Journal of Logic Programming, Vol. 44(1-3), July/August 2000.

[11] N. E. Fuchs. Specifications are (preferably) executable. IEE/BCS Software Engineering Journal, 7(5):323-334, 1992.

[12] Ivar Jacobson, Grady Booch, and James Rumbaugh. The Unified Software Development Process. Addison-Wesley, 1999.

[13] A.C. Kakas, Bert Van Nuffelen, and Marc Denecker. A-system : Problem solving through abduction. In B. Nebel, editor, Proceedings of the Seventeenth International Joint Conference on Artificial Intelligence, pages 591-596. International Joint Conferences on Artificial Intelligence, Inc. and American Association for Artificial Intelligence, 2001.

[14] Craig Larman. Applying UML and Patterns: An Introduction to Object-Oriented Analysis and Design. Prentice Hall, 1997.

[15] Michael Leushel. The ECCE partial deduction system and the dppd library of benchmarks. Obtainable via http://www.ecs.soton.ac.uk/ mal.

[16] James Rumbaugh, Ivar Jacobson, and Grady Booch. The Unified Modeling Language Reference Manual. Addison-Wesley, 1998.

[17] Eric Steegmans, Pieter Bekaert, Frank Devos, Jan Dockx, Bart Swennen, and Stefan Van Baelen. Object-Oriented Analysis. Course Notes, Department of Computer Science, K.U.Leuven, 2001.

[18] Eric Steegmans, Johan Lewi, M. D'Haese, Jan Dockx, D. Jehoul, Bart Swennen, Stefan Van Baelen, and P. Van Hirtum. EROOS Reference Manual. Version 1.0. Report CW 208, Department of Computer Science, K.U.Leuven, Leuven, Belgium, January 1995.

[19] Stefan Van Baelen, Johan Lewi, Eric Steegmans, and Bart Swennen. Constraints in object-oriented analysis. In S. Nishio and A. Yonezawa, editors, Object Technologies for Advanced Software, Lecture Notes in Computer Science, Vol. 742, pages 393-407. Springer-Verlag, Berlin, 1993. ISBN 3-540-57342-9.

[20] Stefan Van Baelen, Johan Lewi, Eric Steegmans, and H. Van Riel. EROOS : An Entity-Relationship based Object-Oriented Specification Method. In B. Magnusson G. Heeg and B. Meyer, editors, Technology of Object-Oriented Languages and Systems TOOLS \%, pages 103-117. Prentice-Hall, Hertsfordshire, UK, 1992.

[21] A. Van Gelder, K.A. Ross, and J.S. Schlipf. The Well-Founded Semantics for General Logic Programs. Journal of the ACM, 38(3):620-650, 1991. 